\title{
Limited Systemic Exposure with Topical Glycopyrronium Tosylate across Multiple Studies in Healthy Volunteers and Patients with Primary Axillary Hyperhidrosis
}

D. M. Pariser', E. L. Lain'2, R. Mamelok ${ }^{3}$, J. Drew ${ }^{4}$, D. R. Mould ${ }^{5}$

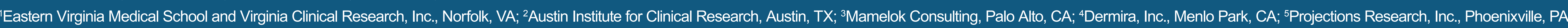

INTRODUCTION

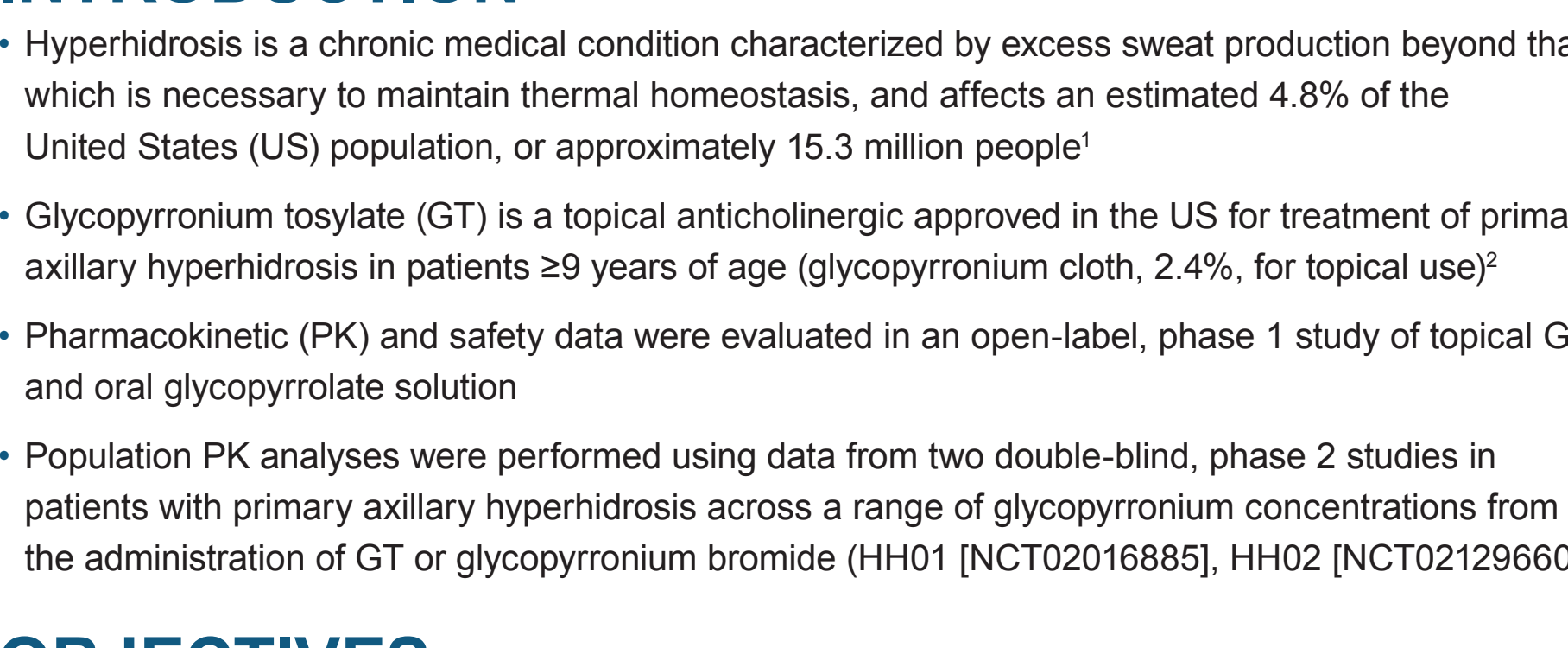

OBJECTIVES

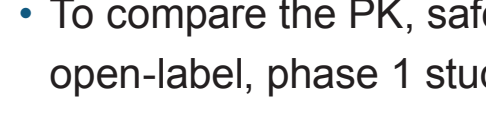

- To assess the relationship of the topical glycopyrronium PK profile to anticholinergic-related adverse
events or feficacy using a population PK and pharmacodynamic model applied to datat from two
phase 2 studies

METHODS

Study Design

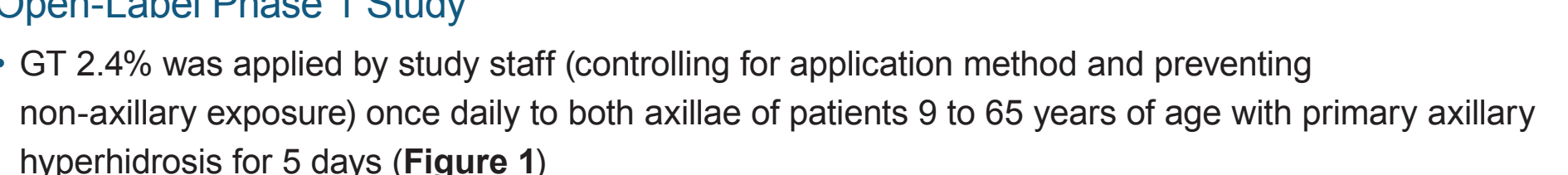

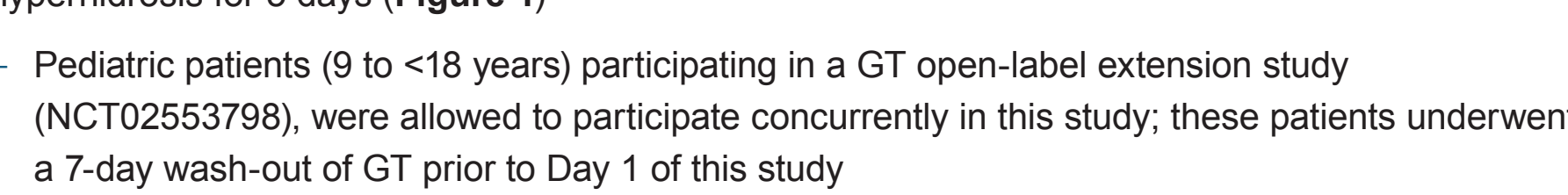

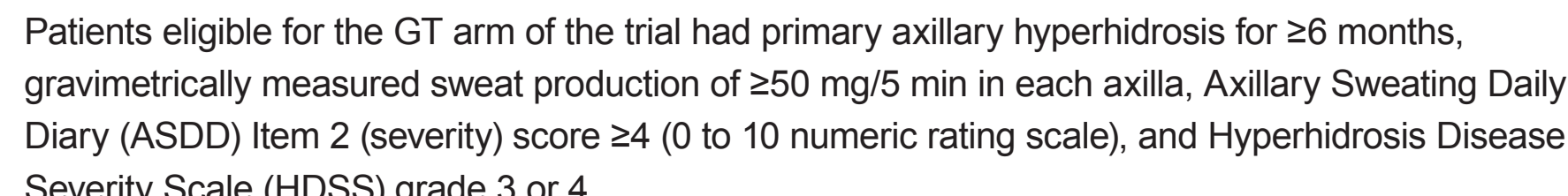

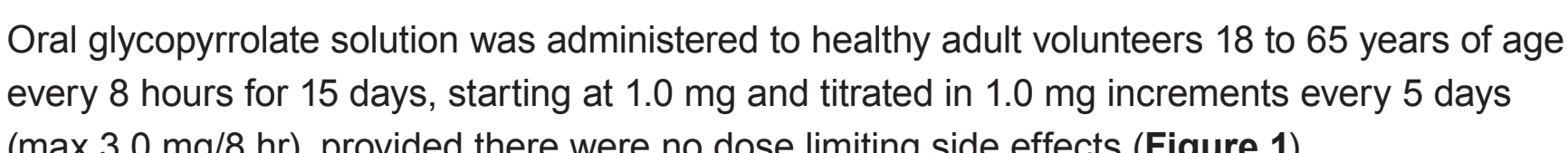

Blood samples were collected on Days $1-5$ in patients treated with $G T$ and Days $1,5,10$, and 15
those adminisistered with oral glycopyrrolate

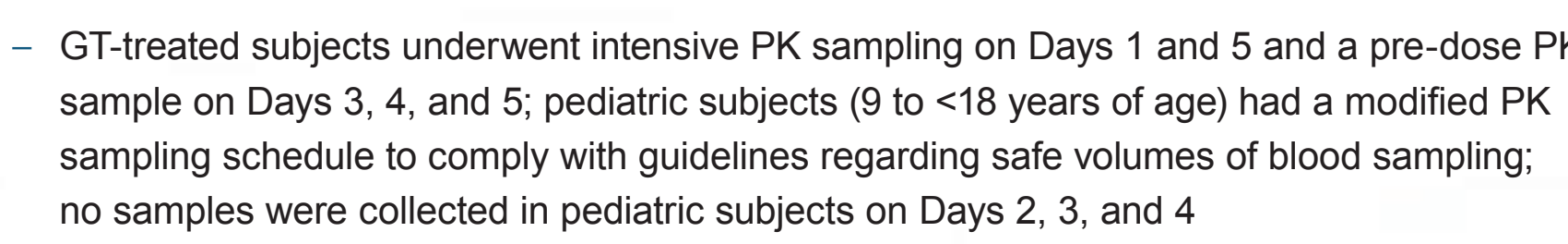

Noncompartmenterteated sujjects underwent intensive PK sampling on Days $1,5,10$, and 15

- Adverse events (AEs) were recorded throughout the study

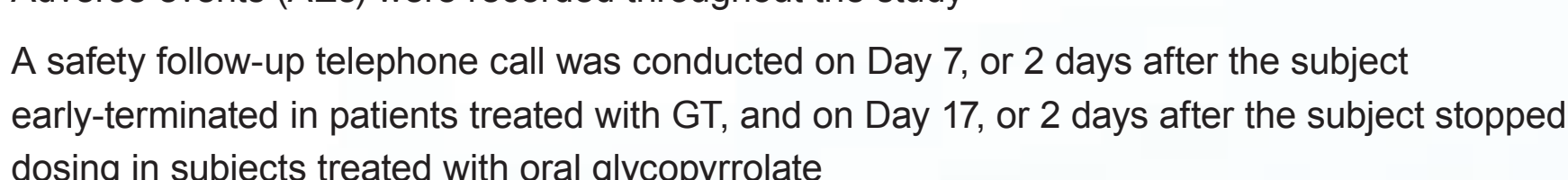

The PK eval
collected

-Concentration values excluded from analysis were any PK samples with
- Detectable concentrations of flycopyrronium in pre--dose samples on Day 1 , and

Plasma concentration values 23 standard devi

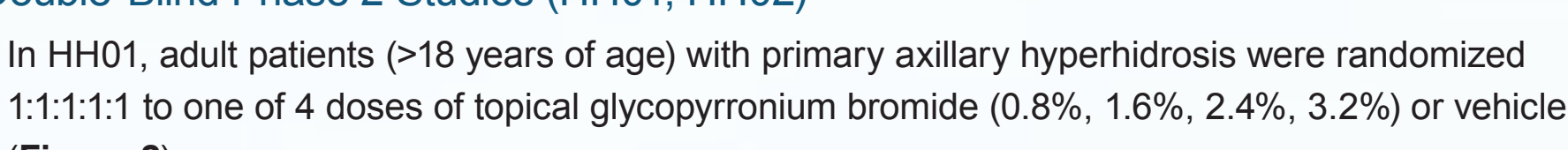

(Figure 2)

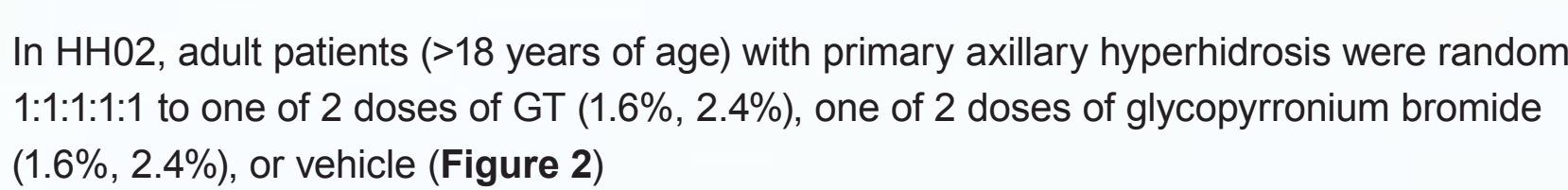

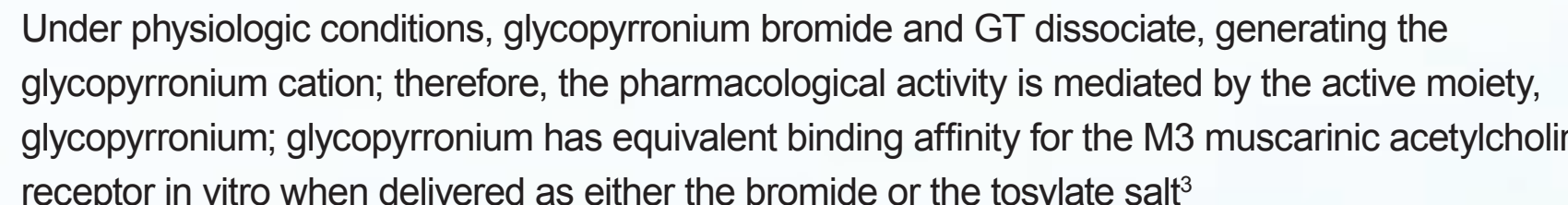

follow-up
. PK data were collected from a subgroup in each study, and AEs and efficacy data were recorded

- In both studies, intensive blood sampling occurred on Days 1 -2
Days 15-16; additional sampling occurred in subsequent weeks

PK data from these phase 2 studies informed a population PK model (NONMEM version 7.2 .0 l con
PLC, Dublin, Ireland) from which exposure metrics were used to assess the relationship between

Figure 1. Phase 1 Open-Label Study Design

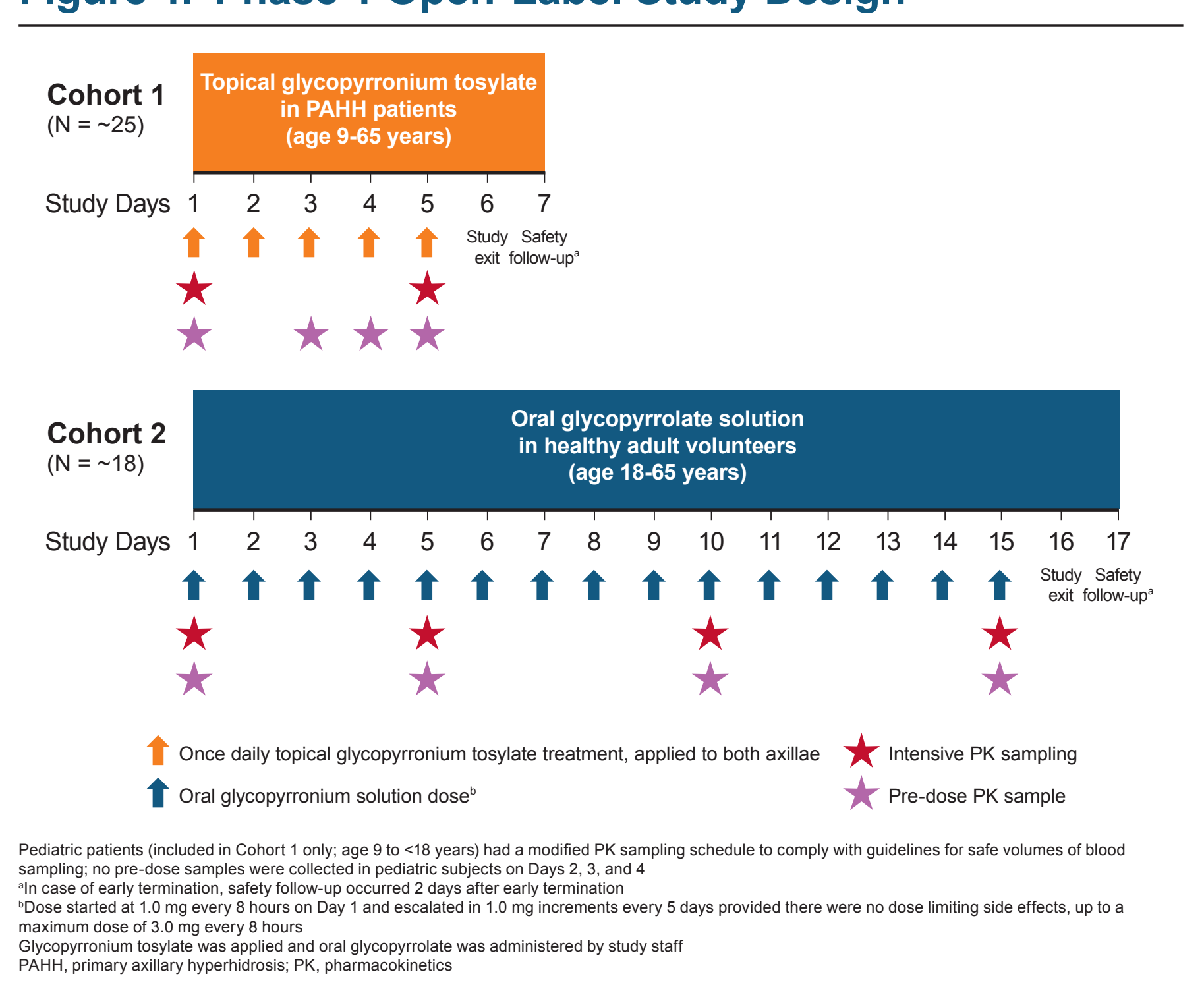

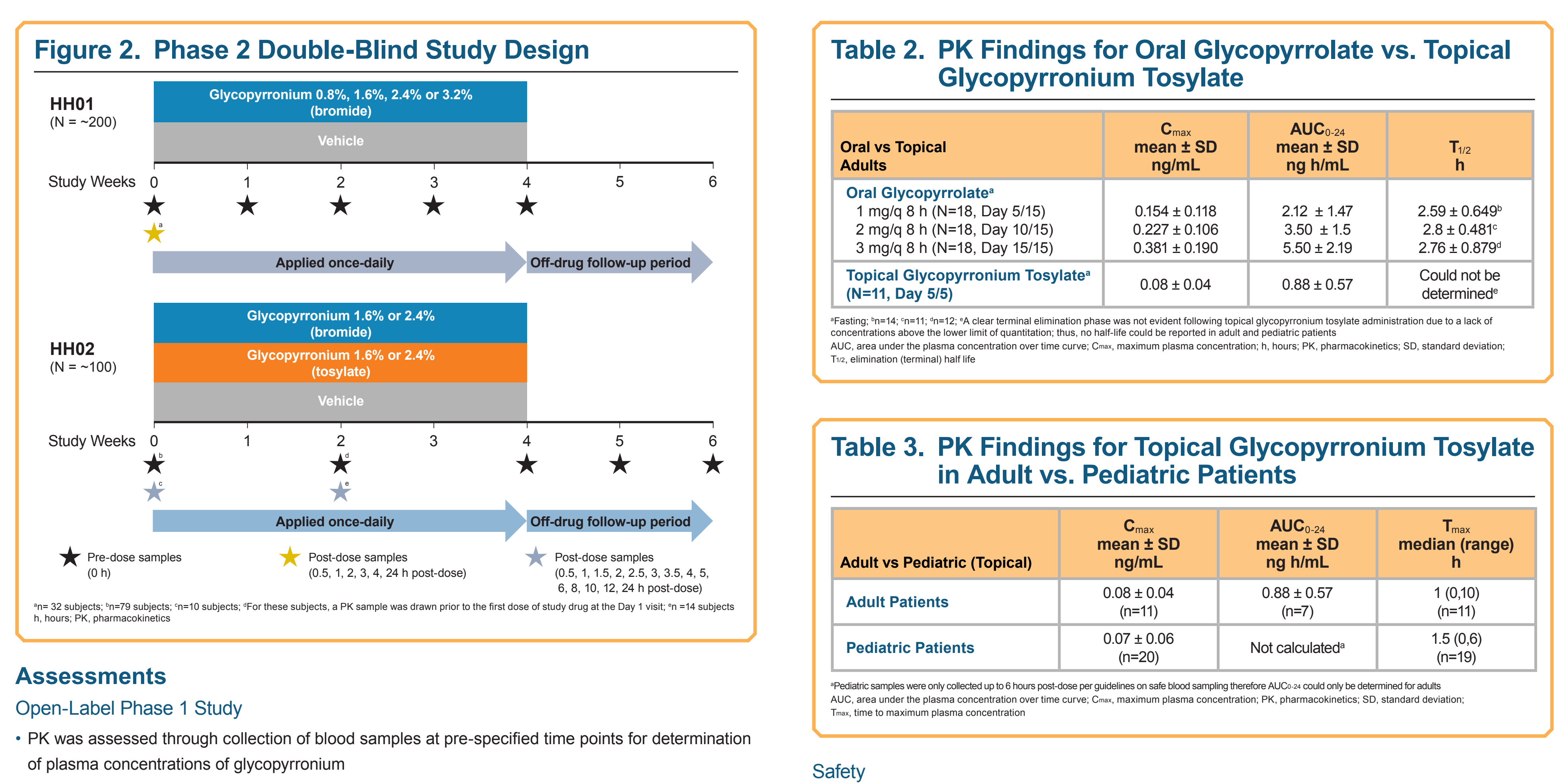

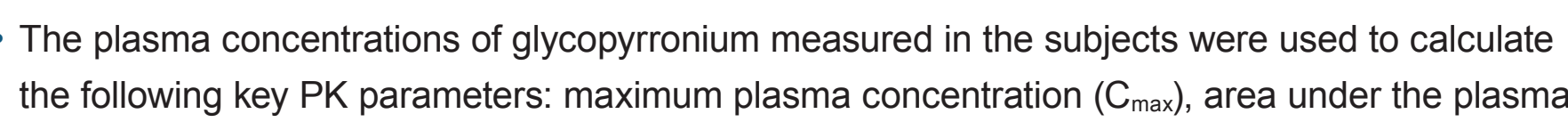

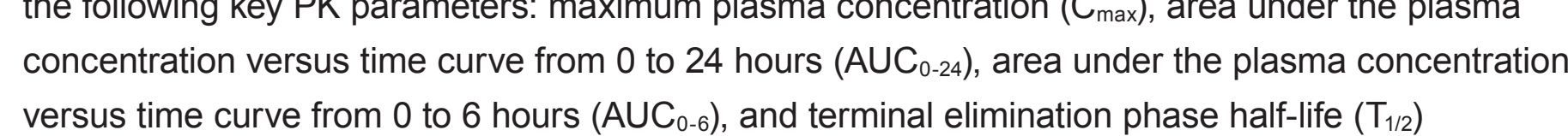

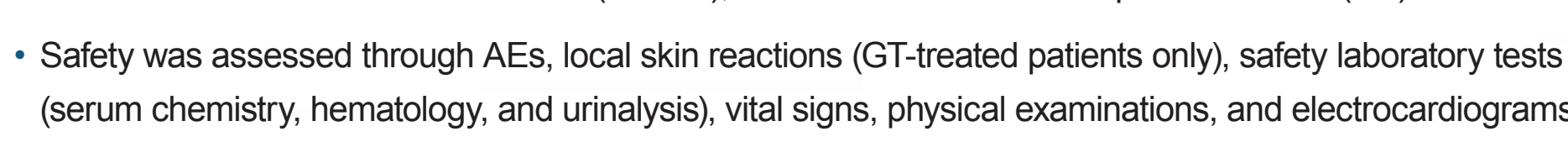

Population PK Analysis of Double-Blind Phase 2 Studies (HHO1, HHO2)

Population PR databasa: all concentration datat from the
HHOL were pooled into a single NONMEM database

- Population PK AE database: includded information on glycopyrronium exposure and the most

Three categories of AEs were defined as

2. Dry mouth, vision blurad
decreased, dry tongue

A

- The worst reported grade was captured using a numericial code ( 0 for no events, 1 for mild,
2 for moderate and 3 for severe) for all patients (including those randomized to the venicle arm) - Population PK PD database: included information on glycopyronium exposure and multiple

RESULTS

Open-Label Phase 1 Study

he phase 1 study, 11 adult (mean age 26 years, $63.6 \%$ female) and 20 pediatric patients

(mean age 14.8 years, $65.0 \%$ female) received topic
$88.9 \%$ male) received oral glycopyrrolate (Table 1 )

Table 1. Phase 1 Open-Label Subject Disposition and
Baseline Characteristics

\begin{tabular}{|c|c|c|c|}
\hline Characteristic & $\begin{array}{c}\text { Topical } \\
\text { Glycopyrronium } \\
\text { Tosylatee } \\
\text { Adults } \\
\text { N=11 }\end{array}$ & 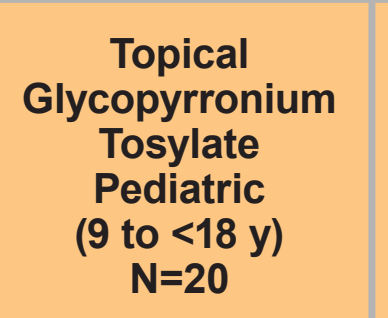 & $\begin{array}{c}\text { Oral } \\
\text { Glycoporrolate } \\
\text { Adults } \\
\mathrm{N}=18\end{array}$ \\
\hline 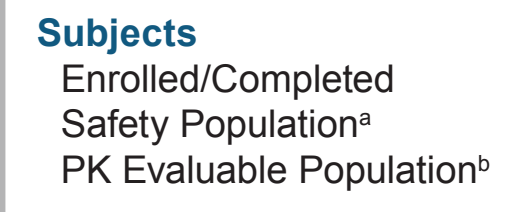 & $\begin{array}{c}11111 \\
11 \\
11\end{array}$ & $\begin{array}{l}20120 \\
20 \\
20\end{array}$ & $\begin{array}{c}18118 \\
18 \\
18\end{array}$ \\
\hline $\begin{array}{l}\text { Age } \\
\text { Mean (SD) } \\
\text { Min, Max }\end{array}$ & $\begin{array}{c}26.0(8.92) \\
18,49\end{array}$ & $\begin{array}{c}14.8(1.64) \\
10,17\end{array}$ & $\begin{array}{l}44.0(10.55) \\
18,58\end{array}$ \\
\hline $\begin{array}{l}\text { Gender, } \boldsymbol{n}(\%) \\
\text { Male } \\
\text { Female }\end{array}$ & $\begin{array}{l}4.436 .4) \\
7(63: 6)\end{array}$ & $\begin{array}{l}7(355.0) \\
13(65 .)\end{array}$ & $\begin{array}{l}16(88.9) \\
2(11.1)\end{array}$ \\
\hline 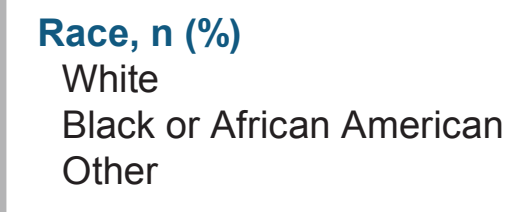 & $\begin{array}{l}9(81.8) \\
2(18.2) \\
0.2)\end{array}$ & 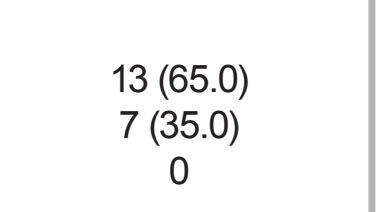 & 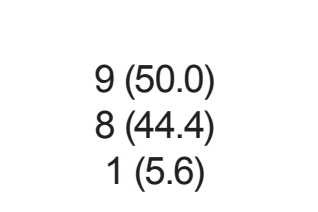 \\
\hline $\begin{array}{l}\text { Ethnicity, n (\%) } \\
\text { Hifspanic or Latino } \\
\text { Not Hispanic or Latino }\end{array}$ & $\begin{array}{l}2(18.2) \\
9(81.8)\end{array}$ & $20(100.0)$ & $\begin{array}{l}2(111) \\
16(88.9)\end{array}$ \\
\hline Weight (kg), mean (SD) & $87.8(27.7)$ & $67.2(16.9)$ & $80.2(9.8)$ \\
\hline BMI (kg/m²), mean (SD) & $29.4(6.3)$ & 23.9 (5.6) & 27.2(2.4) \\
\hline
\end{tabular}

PK Findings

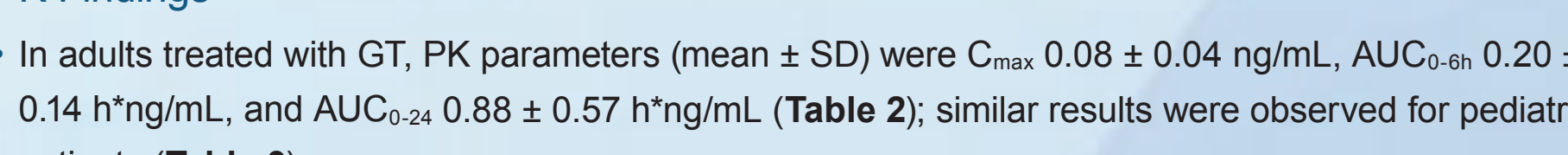

For adults receiving oral glycopyrrolate 1,2 , and $3 \mathrm{mg}$, respectively, mean $\pm \mathrm{SD}$ PK parameters
were $\mathrm{C}_{\max } 0.15 \pm 0.12,0.23 \pm 0.11$, and $0.38 \pm 0.19 \mathrm{ng} / \mathrm{mL}$, and $\mathrm{AUC} \mathrm{C}_{242} 2.12 \pm 1.47,3.50 \pm 1.50$, and
Safety

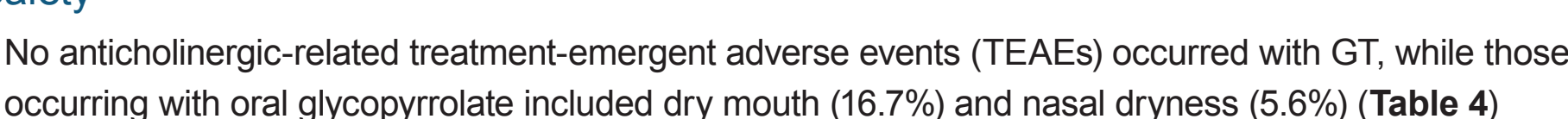

No treatment related TEAEs were reported with GT (Table 4)

Table 4. Safety Findings (Topical Glycopyrronium
Tosylate versus Oral Glycopyrrolate)

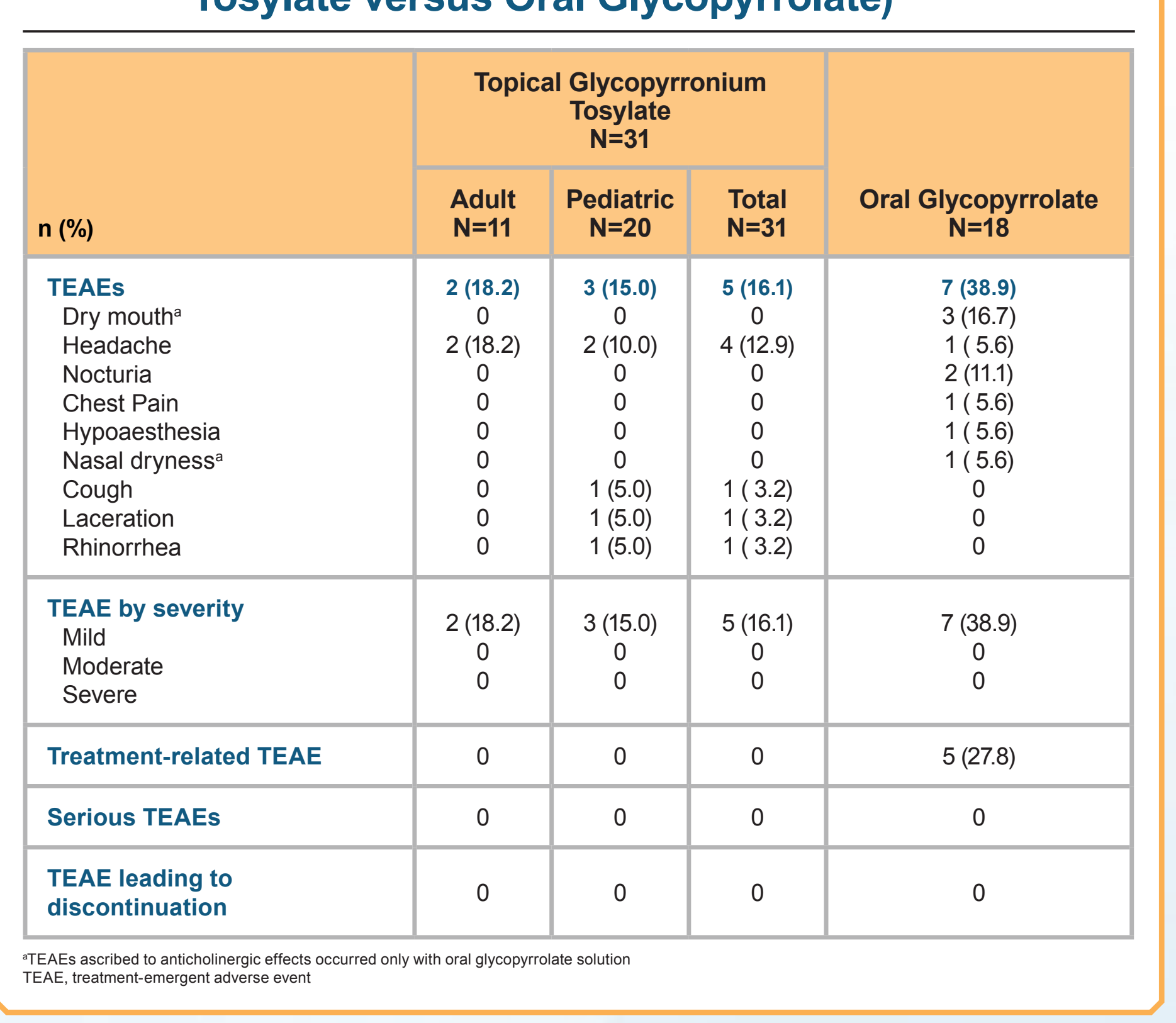

Double-Blind Phase 2 Studies (HH01, HH02)

Subject Demographics and Baseline Characteristics
- In the population PK analysis, 985 PK samples from 108 patients (mean age 32.6 years,
$555 \%$ matel

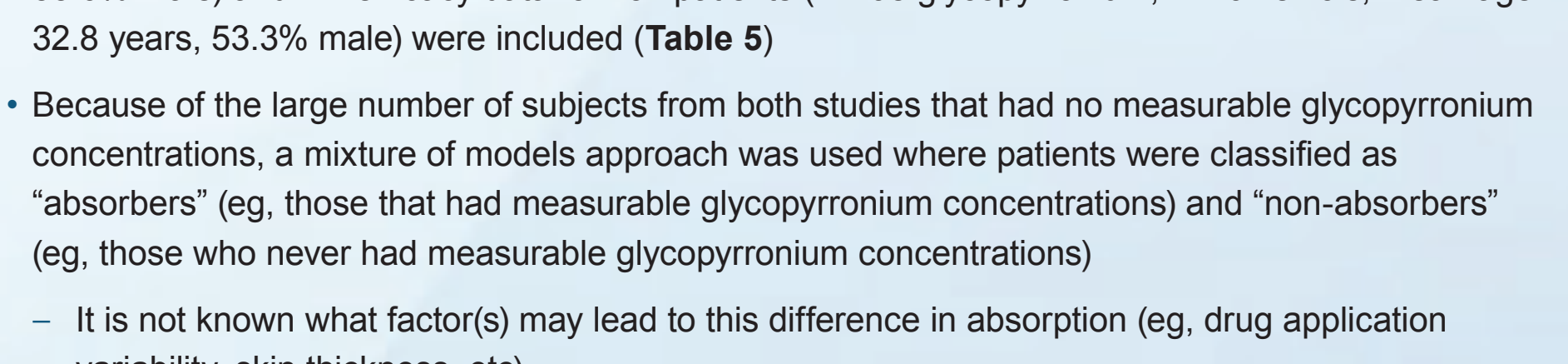

- It is not known what factor(s)
variability, skin thickness, etcos
Table 5. Phase 2 Double-Blind Subject Demographics

\begin{tabular}{|c|c|c|}
\hline Characteristic & $\begin{array}{c}\text { PK Database } \\
N=108\end{array}$ & $\begin{array}{l}\text { PK AE \& PD Database } \\
N=137\end{array}$ \\
\hline \multicolumn{3}{|l|}{ Demographics } \\
\hline $\begin{array}{l}\text { Age } \\
\text { Mean (SDD) } \\
\text { Min, Max }\end{array}$ & $\begin{array}{l}3226(1.6) \\
\text { 18.72 }\end{array}$ & $\begin{array}{c}32.8(11.2) \\
18,72\end{array}$ \\
\hline $\begin{array}{l}\text { Gender, } n \text { (\%) } \\
\text { Malemale } \\
\text { Female }\end{array}$ & 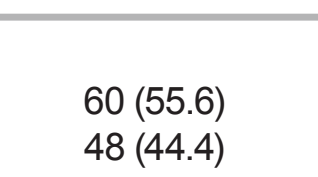 & $\begin{array}{l}73(53.3) \\
64(46.7)\end{array}$ \\
\hline $\begin{array}{l}\text { Race, } n \text { (\%) } \\
\text { Caucasian } \\
\text { Black } \\
\text { Asian }\end{array}$ & $\begin{array}{l}94(87.0) \\
10(0.9) \\
1(0.9)\end{array}$ & $\begin{array}{l}121(88.3) \\
12(8.8) \\
1(0.7)\end{array}$ \\
\hline $\begin{array}{l}\text { Other } \\
\text { Weight (ko) mean (SD) }\end{array}$ & $\frac{3(2.8)}{841126.6}$ & $\frac{3(2.2)}{834.222)}$ \\
\hline BMI (kg/m / $\left.\mathrm{m}^{2}\right)$, ean (SD) & $28.4(6.2)$ & $28.0(6.0)$ \\
\hline \multicolumn{3}{|l|}{ Other Characteristics } \\
\hline 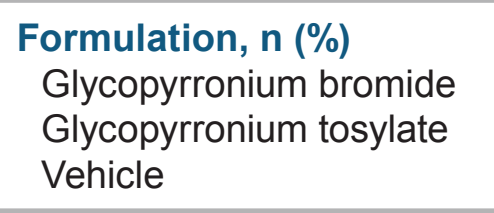 & 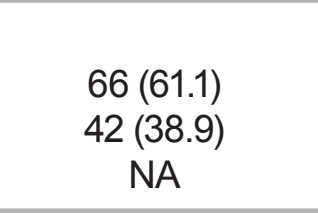 & 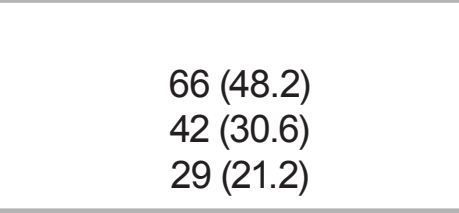 \\
\hline 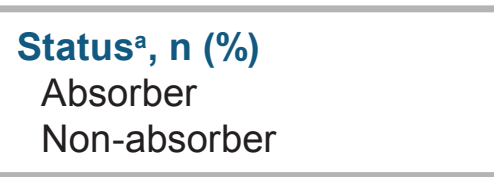 & 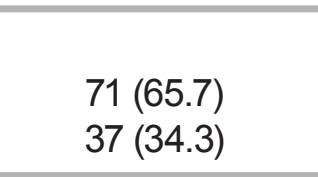 & NA \\
\hline
\end{tabular}

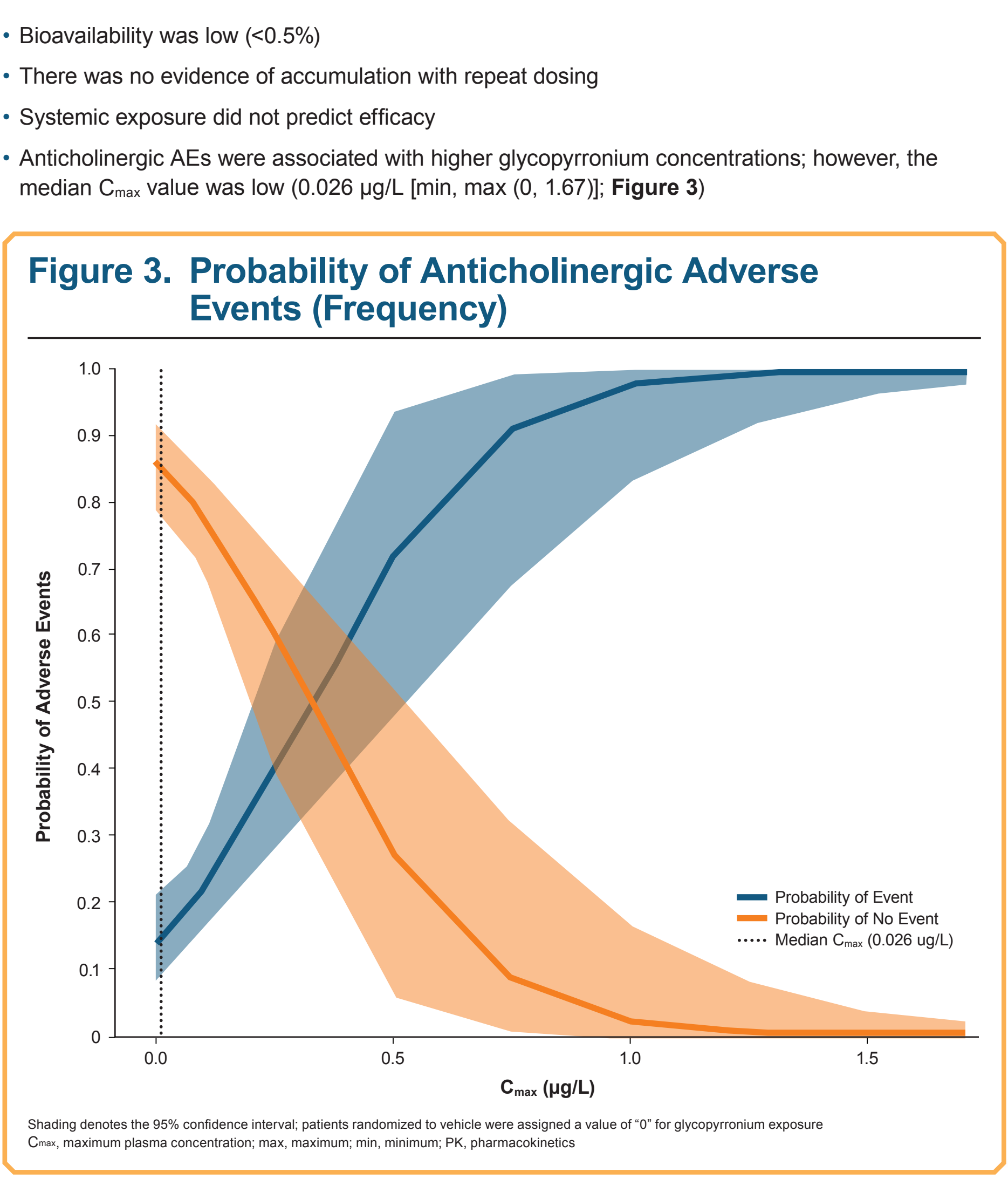

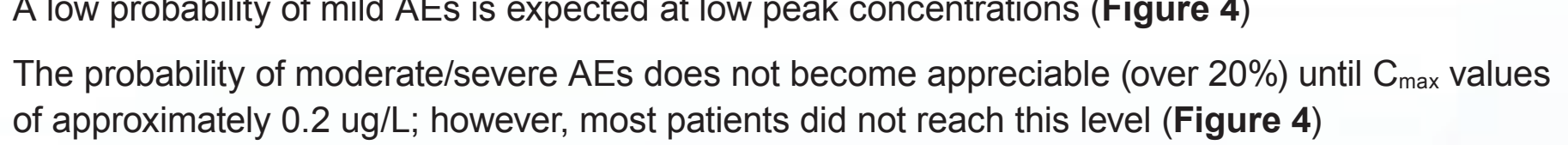

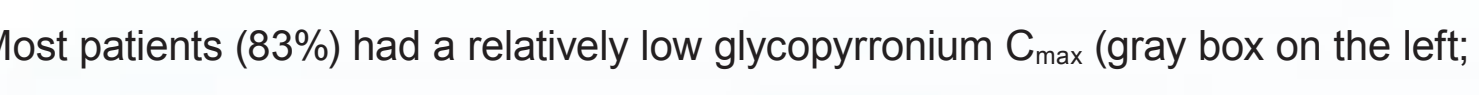

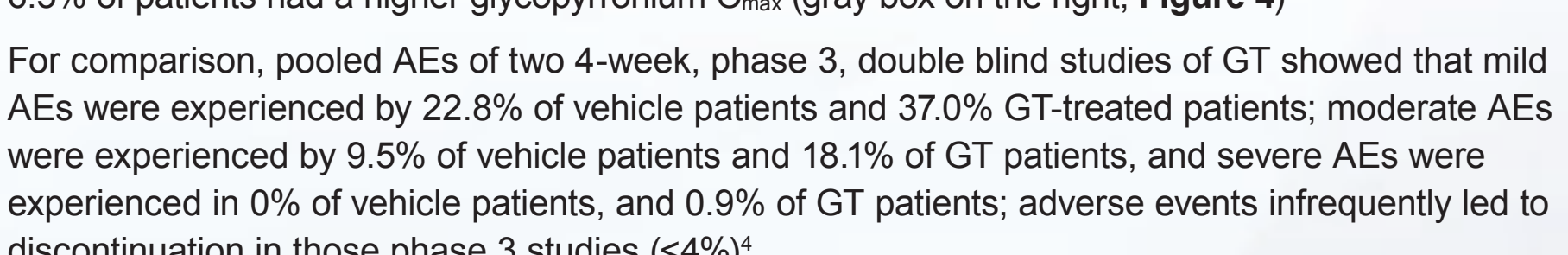

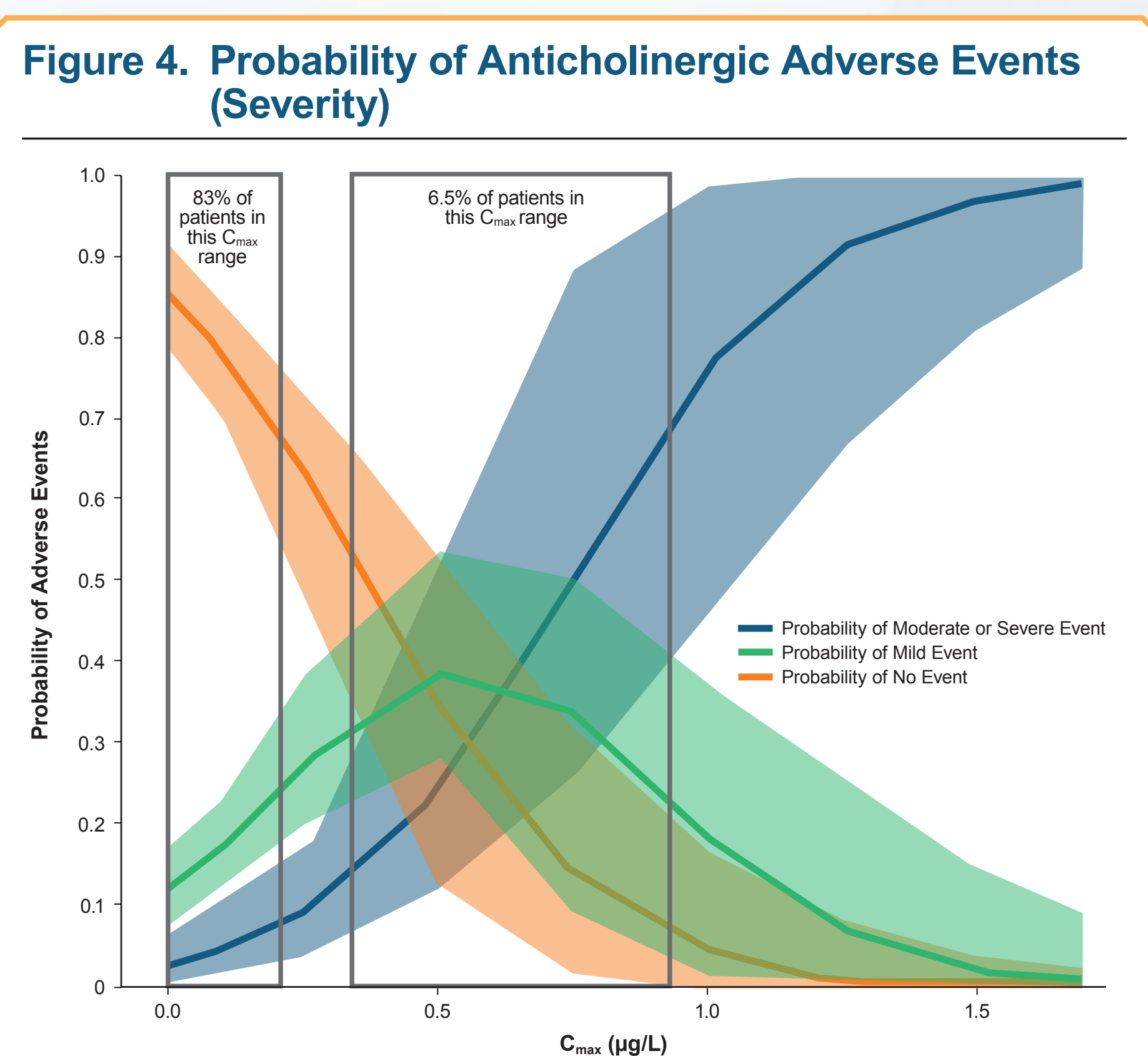

CONCLUSIONS

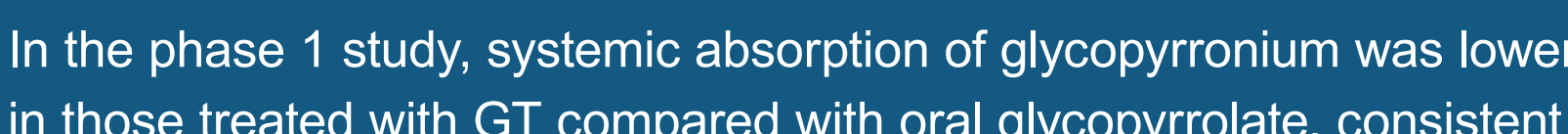

in those treated with GT compared with oral glycopyrrolate, consistent
with the lack of anticholinergic AEs observed with GT in this study

Exposure-response models suggest that the probability of AEs

increases in frequency and severity with increasing glycopyrroniun
$\mathrm{C}_{\max }$ while efficacy may be mediated locally versus systemically

PK parameters of GT indicate lim
of AEs with proper administration

Consistent with these PK modeling results from Phase 2 data, mos

REFERENCES

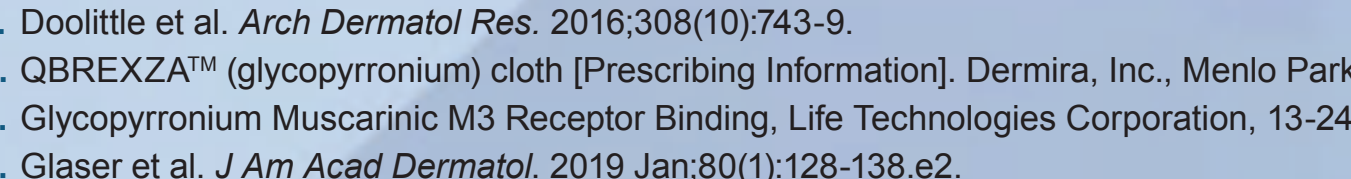

ACKNOWLEDGEMENTS

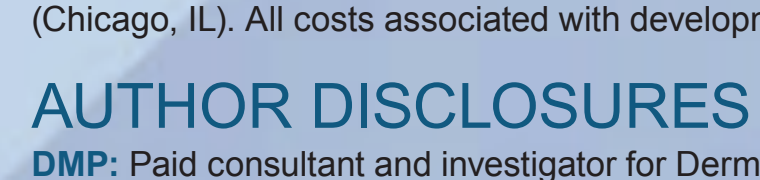

